\title{
PERCEPCIÓN SOBRE RIESGO AL CAMBIO CLIMÁTICO COMO UNA AMENAZA PARA LA SALUD HUMANA, TAGANGA, SANTA MARTA, 2014
}

\author{
Alexander Salazar-Ceballos ${ }^{\underline{1}}$ \\ Nataly Freyle ${ }^{2}$ \\ Germán Tamara $\mathrm{3}^{\underline{3}}$ \\ Lídice Álvarez-Miño ${ }^{4}$
}

Recibido el 23 de abril de 2015, aprobado el 28 de agosto de 2015 y actualizado el 18 de mayo de 2016

DOI: 10.17151/luaz.2016.43.6

\section{RESUMEN}

La Organización Mundial de la Salud considera el cambio climático o calentamiento global un problema de salud pública. De esta manera, se hace necesario conocer la percepción del riesgo al cambio climático en las diferentes comunidades para proponer acciones de adaptación y mitigación al cambio climático; pero esa percepción del riesgo puede variar en las diferentes poblaciones. El objetivo del presente trabajo fue observar la percepción de riesgo sobre el cambio climático como una amenaza para la salud humana en Taganga, Santa Marta, 2014. A nivel metodológico se encuestaron 484 personas de la comunidad de Taganga, mediante un muestreo por conglomerado. Se les aplicó el instrumento validado, a nivel internacional y nacional, mediante el alfa de Cronbach y el coeficiente de Kuder-Richardson 20. Entre los índices se aplicó la correlación de Spearman. Como resultados se observó una correlación estadísticamente significativa entre las acciones políticas frente al cambio climático y el índice de percepción de riesgo; y también entre el índice de voluntad para actuar y los índices de percepción de riesgo, conocimiento sobre los efectos en la salud por causa del cambio climático y las acciones políticas frente al cambio climático. En este estudio se concluye que existe una asociación entre el índice de voluntad para actuar y la percepción de 
riesgo, conocimiento sobre los efectos en la salud por el cambio climático y las acciones políticas frente al cambio climático; igualmente, una asociación entre las acciones políticas frente al cambio climático y la percepción de riesgo.

\title{
PALABRAS CLAVE
}

Cambio climático, riesgo, salud pública, dióxido de carbono, estadísticas no paramétricas.

\section{PERCEPTION OF RISK TO CLIMATE CHANGE AS A THREAT TO HUMAN HEALTH, TAGANGA, SANTA MARTA, 2014}

\begin{abstract}
The World Health Organization considers climate change or global warming a problem of public health. Thus it is necessary to know the perception of risk to climate change in different communities to generate actions for adaptation and mitigation to climate change; but that perception of risk may vary in different populations. The objective of this study was to observe the risk perception on climate change as a threat to human health in the town of Taganga, Santa Marta, 2014. In methods, it was surveyed 484 people in Taganga, through a cluster sampling. It was applied the validated instrument, internationally and nationally, through Cronbach's alpha and Kuder-Richardson formula 20. Spearman's correlation was applied. In results, statistically significant correlation was observed between political action on climate change and risk perception index; and also between the index will to act and indices of risk perception, knowledge about the health effects due to climate change and political action on climate change. This study concludes that was observed an association between the index of willingness to act and risk perception, knowledge about the health effects of climate change and political action on climate change; also an association between political action on climate change and risk perception.
\end{abstract}




\section{KEY WORDS}

Climate change, risk, public health, carbon dioxide, statistics nonparametric.

\section{INTRODUCCIÓN}

La Organización Mundial de la Salud considera el cambio climático o calentamiento global un problema de salud pública (WHO, 2009). La evidencia científica ha demostrado que el actual cambio climático es consecuencia principal de las actividades de quema de combustibles fósiles, las cuales generan dióxido de carbono, $\mathrm{CO} 2$, el principal gas del efecto invernadero y por consiguiente del cambio climático, y estos gases afectan la salud humana (IPCC, 2007). Se define cambio climático a una variación estadísticamente significativa en la media del estado del tiempo por un tiempo prolongado, específicamente décadas; para enfrentar el cambio climático y disminuir sus efectos sobre la salud humana se han establecido estrategias de adaptación y mitigación; así, se ha definido la adaptación como las acciones que se toman, generalmente por los ciudadanos, para reducir la carga de los efectos adversos sobre la salud; y la mitigación se ha definido como las estrategias, políticas, para reducir la emisión de los gases del efecto invernadero. A pesar de que se conocen estas estrategias son pocos los países, tanto desarrollados como en vía de desarrollo, que han generado planes nacionales reales y eficaces, es decir se ha concebido como una obligación moral, para enfrentar el cambio climático (WHO, 2003).

Algunos de los efectos del cambio climático sobre la salud pública son el aumento del dengue (Hales et al., 2002; Johansson, Cummings y Glass, 2009; Rohanni, 2009; Desclouxet al., 2012) y la malaria (Olson et al., 2009; Caminade et al., 2014) que son enfermedades transmitidas por vectores; el cólera (Lama et al., 2004) y la salmonella (Haines et al., 2006; Akil, Ahmad y Reddy, 2014; Franchini y Mannuccio, 2015). También, las olas de calor aumentan la tasa de mortalidad; 
además, se han observado conflictos sobre los suministros de alimentos y agua (Haines et al., 2006). En general, el aumento en la temperatura media de la tierra tendrá efectos extremos sobre las lluvias, la contaminación medioambiental y otras enfermedades como las psiquiátricas (Haines et al., 2006; Franchini y Mannucio, 2015). De esta manera, los impactos del cambio climático tendrán mayor efecto sobre los más desfavorecidos, quienes a su vez son los que menos han contribuido a esta amenaza, aumentando la inequidad en salud con efectos adversos en los determinantes sociales de salud sobre los pobres en los países subdesarrollados (Oloukoi, Bob y Jaggernath, 2014).

El conocimiento de la percepción del riesgo de una amenaza como el cambio climático, debe conducir a la toma de decisiones políticas para mitigar y adaptarse a los efectos del mismo, pero dicha percepción puede variar en las diferentes poblaciones (Taylor, Dessai y Bruine de Bruin, 2014). En las encuestas nacionales en Estados Unidos, Malta y Canadá se ha observado que las personas relacionan el cambio climático con las altas temperaturas y con efectos sobre la salud (Leiserowitz, 2006; Akerlof et al., 2010; DeBono, Vincenti y Calleja, 2010). Entre los efectos sobre la salud, se ha observado que las personas relacionan el cambio climático con el aumento de enfermedades como el dengue, la malaria y el cólera (Rawlins et al., 2007; Akerlof et al., 2010). Pero también, se ha observado que las personas están menos preocupadas por el cambio climático y no creen que sea consecuencia de las actividades humanas (Taylor et al., 2014).

En Colombia son pocos los estudios realizados en relación con la percepción del riesgo al cambio climático y su impacto sobre la salud humana. Recientemente se publicó un trabajo donde se observó que los estudiantes universitarios de Santa Marta, Colombia, conforme avanzaban en sus estudios tenían una percepción del riesgo mayor de los efectos del cambio climático sobre la salud pública (SalazarCeballos et al., 2014). Otros trabajos, si bien exploran la percepción del riesgo al cambio climático, no se han enfocado en el impacto de esta amenaza sobre la salud pública (Echeverri, 2010; Pinilla-Herrera et al., 2012); otro trabajo abordó la gestión del riesgo del cambio climático para la preservación de los ecosistemas y un desarrollo sustentable (Aguirre, Gallego y Cano, 2012). En el presente trabajo el riesgo será definido como: la probabilidad de que se produzca un resultado adverso, o como un factor que aumenta esa probabilidad (WHO, 2002). 
De esta manera, se hace necesario conocer la percepción del riesgo al cambio climático en las diferentes comunidades, para proponer medidas para la adaptación al cambio climático que reduzcan el impacto sobre la salud humana. El objetivo del presente trabajo fue observar la percepción de riesgo sobre el cambio climático como una amenaza para la salud humana en la población de Taganga, Santa Marta, 2014.

\section{MÉTODOS}

Tipo de estudio: de correlación y corte transversal.

Taganga: políticamente, Taganga, latitud N 11016'10,18" y longitud O 74011'24,72", es una comuna y pertenece a la ciudad de Santa Marta, latitud N 11014 '30,99" y longitud O 74012'19". Tiene cerca de tres mil habitantes y es una comunidad que basa su economía principalmente en la pesca artesanal y el turismo.

La ciudad de Santa Marta es endémica para la transmisión del virus del dengue; en estudios previos se observó que en años epidémicos, entre 2007 y 2013, el dengue aumentó desde el mes de junio hasta diciembre, siendo septiembre el mes de mayor incidencia; y se observó asociación estadística entre la precipitación pluvial y la incidencia de dengue (Salazar-Ceballos y Álvarez-Miño, 2014). Por administración política, los casos de dengue de Taganga se analizan en conjunto con la ciudad de Santa Marta, es decir no existe reporte sectorizado de Taganga de acceso al público.

El instrumento que se aplicó está basado en el publicado por DeBono et al. (2010) y consta de cinco índices (ver Anexo): 
- A1, conocimiento sobre los factores que contribuyen al calentamiento global (cambio climático). Para las encuestas se utilizó el término "calentamiento global", término con el cual los ciudadanos están más familiarizados que con "cambio climático" (Leiserowitz et al., 2014). En éste se consideran factores como el uso de vehículos, plaguicidas, teléfonos celulares, entre otros aspectos.

- A2, percepción de riesgo. Este índice es acerca del conocimiento del impacto del cambio climático sobre el modo de vida de las personas. Indaga sobre la probabilidad de que se disminuya la calidad de vida global y local, que haya escasez de agua, que se aumenten las enfermedades, etc.

- A3, conocimiento sobre los efectos en la salud por causa del calentamiento global, que incluye aspectos como las enfermedades infecciosas, asma, alergias, cáncer, alteraciones cardiovasculares, entre otros.

- A4, acciones políticas frente al calentamiento global, a través de aumento de recursos en acciones de información y educación o el aumento de impuestos a las empresas y a los combustibles, entre otras opciones.

- A5, voluntad para actuar. Este índice es acerca del conocimiento de adoptar medidas, a nivel personal, para reducir los efectos sobre el cambio climático, como por ejemplo utilizar transporte público y/o bicicleta, uso de aires acondicionados, comprar paneles solares, etc.

Los índices A1 y A3 contenían solo respuesta Sí o No. La valoración fue así: un punto fue adicionado por cada respuesta correcta y un punto fue deducido por cada respuesta incorrecta. Los índices A2, A4 y A5 tenían respuestas en escala Likert y valoradas de 1 a 5.

El análisis estadístico de la asociación entre los índices fue realizado a través de la correlación de Spearman; de esta manera, se puede observar asociación entre dos variables ordinales aleatorias, este valor de correlación puede variar entre -1 y 1, el signo no expresa fuerza de asociación, el valor de cero indica que no existe 
asociación, y -1 o 1 indicaría una fuerza de asociación perfecta, pero que no es lo normal (Álvarez-Cáceres, 2007).

Según la revisión bibliográfica a la fecha, esta es la primera vez que el instrumento es aplicado en idioma español y en Colombia. Así, el instrumento fue validado por expertos en elaboración de instrumentos y se realizaron los ajustes sugeridos por los mismos. La confiabilidad interna del instrumento se realizó sobre una muestra de 199 estudiantes de la Universidad del Magdalena, 2014. Para el cálculo del coeficiente alfa de Cronbach y el coeficiente de Kuder-Richardson 20 (KR-20) se utilizó la hoja en excel Reliability Calculator 2 elaborada por Del Siegle (2013). El coeficiente del alfa de Cronbach fue utilizado para medir la consistencia interna, la confiabilidad, entre los ítems del instrumento usando escalas de Likert (ÁlvarezCáceres, 2007); y el coeficiente de Kuder-Richardson 20 (KR-20) fue utilizado para medir la consistencia interna de los ítems con respuestas dicotómicas: "sí" o "no" (Oviedo y Campo-Arias, 2005).

Prueba piloto: se encuestaron cuatro manzanas del barrio Villa Universitaria, latitud N 11013'31,96" y longitud O 74011'21,53", Santa Marta, Colombia, y como resultado se obtuvo un total de 20 encuestas utilizando un muestreo aleatorio por conglomerados.

Muestreo: para el estudio se calculó un tamaño de muestra de 484 personas considerando un 95\% de confianza, un 5\% de error; además, se adicionó un efecto de diseño de 1,2 y un $5 \%$ de falta de respuesta para un total de 484 sujetos. La selección de las personas se hizo a través de la técnica de conglomerados, que consiste en seleccionar aleatoriamente lugares donde haya grupos de personas, de tal forma que se seleccionaron 22 manzanas, a través de un recorrido previamente establecido sobre el mapa de Taganga. En cada manzana, desde la esquina, se contaban dos casas y en la tercera se aplicaba la encuesta a todas las personas que estuvieran dentro de la residencia, independientemente de que fueran vecinos, residentes o invitados, previo consentimiento informado. En caso de que la casa a encuestar estuviera vacía, se incluía la vivienda contigua. La recolección de los datos se realizó entre los meses de julio y agosto de 2014. 


\section{RESULTADOS}

De las 484 personas encuestadas 271, aproximadamente el 56\%, eran mujeres y 213 eran hombres (44\%); con un rango de edad entre los 18 y los 89 años de edad y un promedio de 42 años.

Los resultados del alfa de Cronbach y el coeficiente de KR-20 sobre todos los índices del instrumento se presentan en la Tabla 1.

Tabla 1. Resultados del alfa de Cronbach y el coeficiente de KR-20 sobre todos los índices de percepción de riesgo del cambio climático como una amenaza para la salud humana

\begin{tabular}{|l|c|c|}
\hline & KR20 & Alfa de Cronbach \\
\hline $\begin{array}{l}\text { A1. Conocimiento sobre los factores que contribuyen al } \\
\text { calentamiento global (cambio climático) }\end{array}$ & $0,60^{3}$ & \\
\hline A2. Indice de percepción de riesgo & & $0,77^{\circ}$ \\
\hline $\begin{array}{l}\text { A3. Conocimiento sobre los efectos en la salud por causa } \\
\text { del calentamiento global (cambio climático) }\end{array}$ & $0,40^{\circ}$ & \\
\hline $\begin{array}{l}\text { A4. Acciones políticas frente al calentamiento global (cambio } \\
\text { climático) }\end{array}$ & & $0,71^{\circ}$ \\
\hline A5. Indice de la voluntad para actuar & & $0,71^{\circ}$ \\
\hline
\end{tabular}

a: bueno para propósitos exploratorios. b: buena confiabilidad. c: pobre confiabilidad.

Fuente: elaboración propia. 
Tabla 2. Correlación de Spearman entre los índices percepción de riesgo del cambio climático como una amenaza para la salud humana

\begin{tabular}{|l|c|c|c|c|c|}
\hline & A1 & A2 & A3 & A4 & A5 \\
\hline $\begin{array}{l}\text { A1. Conocimiento sobre los factores } \\
\text { que contribuyen al calentamiento } \\
\text { global (cambio climático) }\end{array}$ & 1 & & & & \\
\hline A2. Indice de percepción de riesgo & $-0,052$ & 1 & & & \\
\hline $\begin{array}{l}\text { A3. Conocimiento sobre los efectos } \\
\text { en la salud por causa del cambio } \\
\text { climático }\end{array}$ & $-0,017$ & 0,037 & 1 & & \\
\hline $\begin{array}{l}\text { A4. Acciones políticas frente al } \\
\text { cambio climático }\end{array}$ & $-0,066$ & $0,154 \mathrm{~b}$ & $-0,021$ & 1 & \\
\hline A5. Indice de la voluntad para actuar & $-0,04$ & $0,166 \mathrm{~b}$ & $-0,103 \mathrm{a}$ & $0,149 \mathrm{~b}$ & 1 \\
\hline
\end{tabular}

a: correlgción es estadisticamente significativg al nivel de 0,05 . b: correlación es estadísticamente significativg al nivel de 0,01 .

Fuente: elaboración propia.

De acuerdo a los resultados en la percepción del riesgo al cambio climático como una amenaza para la salud humana entre los habitantes de Taganga, se realizaron los análisis de asociación entre los ítems por medio de la correlación de Spearman y se observó correlación estadísticamente significativa entre las acciones políticas frente al cambio climático (A4) y el índice de percepción de riesgo (A2); y también entre el índice de voluntad para actuar (A5) y los índices de percepción de riesgo (A2), conocimiento sobre los efectos en la salud por causa del cambio climático (A3) y las acciones políticas frente al cambio climático (A4). No se observó asociación estadísticamente significativa entre el conocimiento sobre los factores que contribuyen al calentamiento global (cambio climático) y alguno de los otros cuatro índices (ver Tabla 2).

\section{DISCUSIÓN}

En este estudio de acuerdo a la percepción suministrada por la comunidad de Taganga, Santa Marta, se observó una asociación entre entre el índice de voluntad para actuar (medidas de adaptación por parte de los ciudadanos) y la percepción de riesgo, asociación entre el conocimiento sobre los efectos en la salud por el cambio climático y las acciones políticas frente al cambio climático; igualmente, 
una asociación entre las acciones políticas (medidas de mitigación) frente al cambio climático y la percepción de riesgo. Y aunque no se observó ninguna asociación entre el conocimiento sobre la contribución del cambio climático y los demás índices, la comunidad de Taganga percibe que tomar medidas de adaptación, por parte de los ciudadanos, y mitigación, por parte de los políticos, contribuirá a disminuir los efectos del cambio climático sobre la salud humana.

El actual cambio climático es causado por la emisión de gases efecto invernadero generados por el hombre, principalmente el $\mathrm{CO}_{2}$ producido por los vehículos automotores y la industria. Este aumento en la temperatura afecta el agua potable disponible para los humanos; así, esta relación entre altas temperaturas y sequía tiene efectos sobre la salud humana. Entre los principales efectos sobre la salud se encuentran: muertes principalmente en niños menores de cinco años por diarreas infecciosas; aumento en la transmisión de enfermedades transmitidas por vectores, como el dengue; las muertes por exceso de calor, principalmente en adultos mayores; finalmente, también las muertes por la desnutrición debido a la falta de alimentos por la sequía. De esta manera, para enfrentar el cambio climático y disminuir sus efectos sobre la salud humana se han establecido estrategias de adaptación y mitigación (WHO, 2003, 2009; Haines et al., 2006; Franchini y Mannuccio, 2015). Acorde con la revisión bibliográfica, éste es uno de los primeros trabajos en Colombia que abordan en conjunto las variables anteriores desde la percepción del riesgo sobre la salud humana en una comunidad, resultados que se analizan a continuación.

Las correlaciones significativas entre las variables obtenidas en este trabajo también fueron similares a las obtenidas en el trabajo de DeBono et al. (2010), del cual se adaptó el instrumento al español, donde se analizó la percepción pública del cambio climático como una amenaza para la salud humana en Malta (DeBono et al., 2010); pero en el presente trabajo no se obtuvo correlación entre las variables índice de percepción del riesgo y conocimiento sobre los efectos en la salud por el cambio climático, como sí se obtuvo correlación en el trabajo de DeBono et al. (2010). La validez del instrumento obtuvo resultados similares a los encontrados por DeBono et al. (2010). Estas similitudes en la correlación entre las percepciones entre las dos regiones, Taganga y Malta, representan un gran interés para el análisis dado que están separadas geográficamente, Taganga en la costa Caribe de Sur América y Malta es un país-isla en el Mediterráneo; pero a nivel de 
enfermedades infecciosas comparten la transmisión del virus del dengue y geográficamente son regiones costeras. La interpretación entre cada correlación se irá analizando según el ítem.

La correlación observada entre la percepción del riesgo y la voluntad para actuar indicaría que percibir el cambio climático como una amenaza, influenciaría a las personas en un cambio del comportamiento para tomar acciones para mitigar o adaptarse a los efectos del cambio climático. Esta correlación también fue obtenida previamente en la percepción pública en Malta (DeBono et al., 2010). Se ha observado que la comunicación del riesgo es una herramienta importante que aumentaría la participación de los ciudadanos en programas de adaptación y mitigación del cambio climático (Niles, Lubell y Haden, 2013).

La correlación observada entre la percepción del riesgo y el conocimiento de los efectos sobre la salud por el cambio climático, se explicaría por las experiencias que tienen los habitantes de Taganga en relación con las épocas de sequía, lo cual ha generado escasez de agua potable y olas de calor que se han presentado en los últimos años y con la convivencia con la transmisión endémica del dengue; y posiblemente por la asociación entre sequía y aumento de casos de diarrea. Estos resultados también fueron similares en la percepción del riesgo al cambio climático en Malta, país que también presenta transmisión endémica de dengue (Debono et al., 2010). Previamente en Santa Marta se determinó el acceso y la calidad del agua para el consumo humano en el área urbana de la ciudad, y se observó que cerca del $95 \%$ de las viviendas tienen servicio de agua domiciliaria pero cerca del $50 \%$ presentaron cortes frecuentes del servicio; y con respecto a la calidad del agua de un total de nueve muestras de agua domiciliar, correspondientes a nueve sitios geográficos diferentes, dos evidenciaron la presencia de coliformes fecales; también, estadísticamente se evidenció que los estratos bajos presentaban más fallas en la prestación del servicio de agua (Álvarez-Miño et al., 2013).

Anteriormente se ha observado que la sequía, además de una disminución del acceso al agua potable, conduce a las comunidades a utilizar agua contaminada conllevando al aumento de enfermedades transmitidas por alimentos y por consiguiente un aumento de los casos de diarrea (El-Fadel et al., 2012). Recientemente en México en el estado de Baja California Sur, en zonas naturales, 
se llevó a cabo un estudio de percepción del riesgo del cambio climático y se observó que cerca del 62\% conocían sobre el cambio climático o de sus consecuencias; cerca del $60 \%$ de las personas estaban preocupadas por el agua dado que habían sufrido de huracanes y por sequía en los ríos, igualmente mostraron preocupación porque los agricultores explotaban los acuíferos y los contaminaban (Olmos-Martínez, González-Ávila y Contreras-Loera, 2013).

La correlación observada en este trabajo entre las acciones políticas frente al cambio climático y la percepción del riesgo y la voluntad para actuar, se explicaría por la percepción de la población acerca de los responsables de la toma de decisiones políticas que conduzcan a la adaptación y mitigación de los efectos sobre la salud por el cambio climático (Taylor et al., 2014); estas correlaciones también fueron encontradas en la encuesta de percepción pública del riesgo al cambio climático en Malta (DeBono et al., 2010). A su vez, con la implementación de políticas se generarían cambios en los comportamientos de las personas para mitigar los efectos del cambio climático. Estas acciones de políticas "verdes" bien pueden ser sembrar, utilizar energías renovables e impuestos para quienes generen combustión de combustibles fósiles (Bostrom et al., 2012; Díaz-Cordero, 2012). Las personas realizarían sus cambios de comportamiento en la obtención de energía más eficiente y menos contaminante, a su vez reducirían las emisiones de $\mathrm{CO} 2$ al hacer uso de medios de transporte como la bicicleta. También se ha observado que las personas con mayor preocupación por los efectos del cambio climático también son las más interesadas por las decisiones políticas al respecto que toman sus gobernantes (Niles et al., 2013).

A nivel de políticas públicas en el Caribe colombiano, se ha observado que principalmente estas acciones están dirigidas hacia la atención de desastres naturales y poco a la prevención, y aunque se han realizado acciones de mitigación como un parque eólico en el departamento de La Guajira su impacto no es significativo en el Caribe colombiano (Guerrero, Flórez y García, 2014). Un reciente análisis del impacto sobre la salud por consecuencias de eventos climáticos extremos observó que, en Colombia durante 2010, Chocó fue el primer departamento con más personas afectadas y el Magdalena el segundo (De la Mata y Valencia-Amaya, 2014), indicando que este último es vulnerable a la amenaza de los eventos extremos del clima. Esta evidencia junto con los resultados del presente estudio sería de utilidad en Taganga, Santa Marta y en el Caribe 
colombiano a la hora de realizar estrategias de comunicación del riesgo y de implementar medidas de adaptación y mitigación al cambio climático.

Los escenarios en Colombia del cambio climático indican que entre los años 2011 y 2040 la temperatura media del aire aumentaría entre 2 y 30C, y entre los años 2070 y 2100 se presentaría un aumento de la temperatura media del aire entre 3 y 40C, en consecuencia se esperaría una reducción de la precipitación en cerca del 30\% en la región Caribe (García et al., 2012; Pabón, 2012). A los escenarios anteriores se le debe sumar que la región Caribe se encuentra entre las zonas con mayor desertificación en Colombia con porcentajes entre 50 y $75 \%$ (Olivero-Verbel, 2011; Vallejo, 2011). Otro factor de interés en la región Caribe es que los niveles de pobreza o pobreza extrema se encuentran en cerca del 57\% (Calderón, Harris y Kirsch, 2015). Además, el Caribe colombiano es la región con más altas tasas de deforestación, y para 2009 y 2010 no fue una región adaptada para soportar eventos climáticos extremos; es de recordar que durante 2010 se presentó el fenómeno de La Niña que afectó a cerca de un millón trescientas mil personas y murieron cerca de 200, este evento fue considerado el peor desastre natural del país (Vallejo, 2011). El IPCC (2007) y la OMS (WHO, 2009) han evidenciado que quienes sufrirán más las consecuencias del cambio climático serán los pobres, y no los países causantes de los gases de efecto invernadero; así, la región Caribe presenta un escenario extremo para la subsistencia de los seres humanos.

La falta de correlación entre el conocimiento de los factores que contribuyen al cambio climático y los demás índices, fue similar al encontrado en el estudio de percepción del riesgo al cambio climático en Malta (DeBono et al., 2010). Estos resultados se explicarían porque el conocimiento de los factores que contribuyen al cambio climático no tiene efectos sobre las políticas, el riesgo, la salud y la voluntad para actuar; o también, implicaría la falta de conocimiento en la relación entre los anteriores índices. En la región Caribe, en las comunidades vulnerables frente a los efectos ambientales del cambio climático, se evidenció que cerca del $50 \%$ reconocían la educación ambiental como un aspecto importante para la mitigación, y en porcentajes inferiores al $1 \%$ se encontraban los aspectos de generación de energías alternativas y proyectos de reforestación (Guerrero et al., 2014). 
La sociedad, y específicamente Colombia y la región Caribe, deberá adoptar estrategias de adaptación y mitigación frente al cambio climático (Posada, 2007). La estrategia de adaptación es generar medidas preventivas para reducir el riesgo de los efectos sobre la salud por el cambio climático, estas intervenciones incluyen: sistemas de vigilancia temprana de las enfermedades infecciosas, sistemas de alerta temprana del clima, sistemas de atención a desastres, educación en salud pública y legislación. Y la estrategia de mitigación está enfocada en reducir las fuentes generadoras de emisiones de gases de efecto invernadero, estas estrategias son a largo plazo porque exigen convenios macro entre los países; en términos técnicos es la adopción del uso de fuentes de energía eficientes, el uso de fuentes de energía renovables y la reforestación (Haines et al., 2006; Semenza et al., 2008). Si bien algunas de estas medidas ya se han adoptado aún su impacto no es significativo.

Desde este trabajo, como una medida de adaptación se sugiere la estrategia de educación de los efectos del cambio climático sobre la salud humana, esta es una estrategia a corto plazo dado que las consecuencias sobre la salud pública ya se están observando; las estrategias de mitigación son a largo plazo y requieren de la buena voluntad política de los gobernantes, específicamente de los países desarrollados quienes son los mayores contaminantes, voluntad política que se dilata mientras el cambio climático avanza.

\section{CONCLUSIONES}

En este trabajo se observó la percepción que tienen los habitantes de Taganga acerca del conocimiento sobre los factores que contribuyen al cambio climático, el riesgo global, el riesgo sobre la salud, las medidas de mitigación por parte de los políticos y las medidas de adaptación por parte de los ciudadanos. 
No se observó ninguna asociación entre el conocimiento sobre la contribución del cambio climático y los demás índices.

La comunidad de Taganga percibe que tomar medidas de adaptación y mitigación contribuirá a disminuir los efectos del cambio climático sobre la salud humana.

En este estudio se observó una asociación entre entre el índice de voluntad para actuar y la percepción de riesgo, conocimiento sobre los efectos en la salud por el cambio climático y las acciones políticas frente al cambio climático; igualmente, se observó una asociación entre las acciones políticas frente al cambio climático y la percepción de riesgo.

\section{AGRADECIMIENTOS}

Los autores agradecen a la Universidad del Magdalena y a la comunidad de Taganga.

\section{POTENCIAL CONFLICTO DE INTERESES}

Los autores declaran no tener conflicto de intereses. Esta investigación fue financiada por la Universidad del Magdalena. 


\section{REFERENCIAS}

- Aguirre, Y.; Gallego, C. y Cano, H.P. (2012). Abordaje metodológico para emprender acciones interinstitucionales e interdisciplinares sobre cambio climático en el departamento de Caldas. Luna Azul, 34, 81-100. Recuperado dehttp://lunazul.ucaldas.edu.co/index.php?option=content\&task=view\&id=697

- $\quad$ Akerlof, K.; DeBono, R.; Berry, P.; Leiserowitz, A.; Roser-Renouf, C.; Clarke, K.L. et al. (2010). Public Perceptions of Climate Change as a Human Health Risk: Surveys of the United States, Canada and Malta. Int J Environ Res Public Health, 7(6), 2559-606.

- $\quad$ Akil, L.; Ahmad, H.A. y Reddy, R.S. (2014). Effects of climate change on Salmonella infections. Foodborne Pathog Dis, 11(12), 974-80. doi: 10.1089/fpd.2014.1802.

- Álvarez-Cáceres, R. (2007). Estadística aplicada a las ciencias de la salud. España: Ediciones Díaz de Santos.

- Álvarez-Miño, L.; Cantillo, K.; Rico, K. y Salazar-Ceballos, A. (2013). Acceso y calidad del agua para el consumo humano en Santa Marta como indicador de inequidad en salud. Rev. Univ. Salud, 15(2), 113-122.

- $\quad$ Bostrom, A.; O'Connor, R.E.; Bohm, G.; Hanss, D.; Bodi, O.; Ekstrom, F. et al. (2012). Causal thinking and support for climate change policies: International survey findings. Glob Environ Change, 22, 210-222.

- $\quad$ Calderón, A.; Harris, J. y Kirsch, P. (2015). Health interventions used by mayor resource companies operating in Colombia. Resources Policy. http://dx.doi.org/10.1016/j.resourpol.2015.02.003 
- Caminade, C.; Kovats, S.; Rocklov, J.; Tompkins, A.M.; Morse, A.P.; ColónGonzález, F.J.et al. (2014). Impact of climate change on global malaria distribution. PNAS, 111(9), 3286-3291.

- DeBono, R.; Vincenti, K. y Calleja, N. (2010). Risk communication: climate change as a human-health threat, a survey of public perceptions in Malta. Eur J Public Health, 22(1), 1-7.

- Del Siegle. (2013). Reliability Calculator 2. School of Education. University of Connecticut. (Creado 09.24.2002 -

- Editado

17/10/2013).

Recuperado

de http://www.gifted.uconn.edu/siegle/research/

instrument\%20reliability\%20and\%20validity/reliability.htm

- De la Mata, D. y Valencia-Amaya, M. (2014). The Health Impacts of Severe Climate Shocks in Colombia. Inter-American Development Bank. Working paper series No. No idb-wp-498. Recuperado de http://publications.iadb.org/bitstream/handle/

11319/6570/The\%20Health\%20Impacts\%20of\%20Severe

\%20Climate\%20Shocks\%20in\%20Colombia.pdf; jsessionid=90EF7BFD0D1569AE6FE1C2F44052B7A6?

sequence $=1$

- $\quad$ Descloux, E.; Mangeas, M.; Eugéne, C.; Lengaigne, M.; Leroy, A.; Tehei, T. et al. (2012). Climate-based models for understanding and forecasting dengue epidemics.PLoS Negl Trop Dis, 6(2), 1470-1473.

- Díaz-Cordero, G. (2012). El cambio climático. Cienc Soc, 37(2), 227-240.

- Echeverri, J.A. (2010). Percepciones y efectos de cambio climático en grupos indígenas de la amazonía colombiana. Folia Amazónica, 19(1), 85-93. 
- El-Fadel, M.; Gahnimeh, S.; Maroun, R. y Alameddine, I. (2012). Climate change and temperature rise: Implications on food- and water-borne diseases. Sci Total Environ, 437, 15-21.

- Franchini, M. y Mannuccio, P. (2015). Impact on human health of climate $\begin{array}{lllll}\text { changes. Eur Intern } & \text { M }\end{array}$ http://dx.doi.org/10.1016/j.ejim.2014.12.008

- García, M., Piñeros, A.; Bernal, F. y Ardila, E. (2012). Variabilidad climática, cambio climático y el recurso hídrico en Colombia. Revista de Ingeniería, 36, 60-64.

- Guerrero, R.; Flórez, J. y García, L. (2014). Estado y prospectiva de las posibilidades de mitigación de impacto del cambio climático en la región Costa Atlántica. Papel Político, 19(1), 121-146.

- Haines, A.; Kovats, R.S.; Campbell-Lendrumb, D. y Corvalan, C. (2006). Climate change and human health: Impacts, vulnerability and public health. Public Health, 120, 585-6.

- Hales, S.; De Wet, N.; Maindonald, J. y Woodward, A. (2002). Potential effect of population and climate changes on global distribution of dengue fever: an empirical model. Lancet, 360(9336), 830-834.

- Intergovernmental Panel on Climate Change (IPCC). (2007). Climate Change 2007: Working Group I: The Physical Science Basis. 9.7 Combining evidence of anthropogenic climate change. Contribution of Working Group I to the Fourth Assessment Report of the Intergovernmental Panel on Climate Change. Solomon, S.; Qin, D.; Manning, M.; Chen, Z.; Marquis, M.; Averyt, K.B.; Tignor, M. y Miller, H.L. (Eds.). Cambridge University Press, Cambridge, United Kingdom and New York, NY, USA. Recuperado dehttp://www.ipcc.ch/publications_and_data/ar4/wg1/en/ch9s9-7.html 
- Johansson, M.A.; Cummings, D.A.T. y Glass, G.E. (2009). Multiyear Climate Variability and Dengue-EI Niño Southern Oscillation, Weather, and Dengue Incidence in Puerto Rico, México, and Thailand: A Longitudinal Data Analysis. PLoS Med, 6(11), e1000168.

- Lama, J.; Seas, C.; León-Barúa, R.; Gotuzzo, E. y Sack, R. (2004). Environmental temperature, cholera, and acute diarrhoea in adults in Lima, Peru. J Health Popul Nutr, 22(4), 399-403.

- Leiserowitz, A. (2006). Climate change risk perception and policy preferences: the role of affect, imagery, and values. Climate Change, 77, 45-2.

- Leiserowitz, A.; Maibach, E.; Roser-Renouf, C.; Feinberg, G. y Howe, P. (2014). Climate change in the American mind: Americans' global warming beliefs and attitudes in April, 2014. Yale University and George Mason University. New Haven, CT: Yale Project on Climate Change Communication.

- $\quad$ Niles, M.; Lubell, M. y Haden, V. (2013). Perceptions and responses to climate policy risks among California farmers. Global Environmental Change, 23, 1752-1760. http://dx.doi.org/10.1016/j.gloenvcha.2013.08.005

- Olivero-Verbel, J. (2011). Colombia: Environmental Health Issues. En Reference Module in Earth Systems and Environmental Sciences, from Encyclopedia of Environmental Health, 740-754, Elsevier.

- Olmos-Martínez, E.; González-Ávila, M. y Contreras-Loera, M. (2013). Percepción de la población frente al cambio climático en áreas naturales protegidas de Baja California Sur, México. Polis, Revista Latinoamericana, 12(35), 459-481.

- Oloukoi, G.; Bob, U. y Jaggernath, J. (2014). Perception and trends of associated health risks with seasonal climate variation in Oke-Ogun region, Nigeria. Health

Place, 25 , 47-55. http://dx.doi.org/10.1016/j.healthplace.2013.09.009 
- Olson, S.; Gangnon, R.; Elguero, E.; Durieux, L.; Guégan, J.F.; Foley, J. y Patz, J. (2009). Links between climate, malaria, and wetland in the Amazon Basin. EID, 15(4), 659-662.

- Oviedo, H.C. y Campo-Arias, A. (2005). Aproximación al uso del coeficiente alfa de Cronbach. Revista Colombiana de Psiquiatría, 34(4), 572-580.

- Pabón, J. (2012). Cambio climático en Colombia: tendencias en la segunda mitad del siglo XX y escenarios posibles para el siglo XXI. Rev Acad Colomb Cienc, 36(139), 261-278.

- Pinilla-Herrera, M.C.; Rueda, A.; Pinzón, C. y Sánchez, J. (2012). Percepciones sobre los fenómenos de variabilidad climática y cambio climático entre campesinos del centro de Santander, Colombia. Ambiente y Desarrollo, 16(31), 25-37.

- Posada, C. (2007). La adaptación al cambio climático en Colombia. Revista de Ingeniería, 26, 74-80.

- Rawlins, S.C.; Chen, A.; Rawlins, J.M.; Chadee, D.D. y Legall, G. (2007). Knowledge, Attitude and Practices Study of the Issues of Climate Change/Variability Impacts and Public Health in Trinidad and Tobago, and St Kitts and Nevis. West Indian Med J, 56(2), 115-1.

- Rohani, P. (2009). The link dengue incidence and El Niño Southern Oscillation. PLoS Med, 6(11), e1000185.

- Salazar-Ceballos, A. y Álvarez-Miño, L. (2014). Asociación entre factores climatológicos y tasa de incidencia del dengue en Santa Marta, Colombia, 2007-2013. Revista Ciencias Biomédicas, 5(1), 41-47. 
- Salazar-Ceballos, A.; Álvarez-Miño, L.; Muñoz-Sánchez, E.P.; CarreñoOrozco, J.D. y Rodríguez-Choles, B.H. (2014). Percepción del riesgo al cambio climático y sus efectos sobre la salud y enfermedades infecciosas en estudiantes universitarios, Santa Marta, Colombia, 2011. Rev Cuid, 5(1), 61322.

- Semenza, J.C.; Hall, D.E.; Wilson, D.J.; Bontempo, B.D.; Sailor, D.J. y George, L.A. (2008). Public perception of climate change. Voluntary mitigation and barriers to behavior change. Am J Prev Med, 35(5), 479-87. http://dx.doi.org/10.1016/j.amepre.2008.08.020

- Taylor, A.; Dessai, S. y Bruine de Bruin, W. (2014). Public perception of climate risk and adaptation in the UK: A review of the literature. Climate Risk Management, 4-5, 1-16. http://dx.doi.org/10.1016/j.crm.2014.09.001

- Vallejo, J. (2011). A Regional Strategy for Water Resource Management in the Caribbean Region of Colombia. Tropical Resources Bulletin, 30, 28-35.

- World Health Organization. (2002). World health report. Reducing Risks, Promoting Healthy Life. Geneva, Switzerland: WHO. Recuperado de http://www.who.int/whr/2002/en/

- World Health Organization. (2003). Methods of assessing human health vulnerability and public health adaptation to climate change. Health and global environmental change. Series No. 1. Lead authors: Kovats, S.; Ebi, K. y Menne, B. Denmark. Recuperado de http://apps.who.int/iris/handle/10665/107538

- World Health Organization. (2009). Protecting health from climate change: connecting science, policy and people. Denmark. Recuperado dehttp://www.who.int/globalchange/publications/reports/9789241598880/en/ 
1. Maestría en Biología. Docente Programa de Medicina, Universidad del Magdalena. Santa Marta, Colombia. alexsal2010@gmail.com. ORCID: 0000-00020708-8792

2. Estudiante del Programa de Medicina, Universidad del Magdalena. Santa Marta, Colombia. natyfreyle@hotmail.com. ORCID: 0000-0003-4365-5325

3. Estudiante del Programa de Medicina, Universidad del Magdalena.plasmager2000@hotmail.com. ORCID: 0000-0002-4627-5793

4. Maestría en Salud Pública. Docente Programa de Enfermería, Universidad del Magdalena. Santa Marta, Colombia. lidice@lycos.com. ORCID: 0000-0002-14149442

Para citar este artículo: Salazar-Ceballos, A.; Freyle, N.; Tamara, G. y ÁlvarezMiño, L. (2016). Percepción sobre riesgo al cambio climático como una amenaza para la salud humana, Taganga, Santa Marta, 2014. Revista Luna Azul, 43, 102$127 . \quad$ Recuperado de http://200.21.104.25/lunazul/index.php?option=com_content\&view=article\&id=195

Esta obra está bajo una Licencia de Creative Commons Reconocimiento CC BY

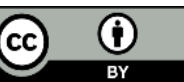




\section{ANEXO}

Encuesta de percepción sobre riesgo al cambio climático como una amenaza para la salud humana, Taganga, Santa Marta, 2014.

[Tomado y adaptado de DeBono et al. (2010)]

Edad:

Sexo: Mujer Hombre

Estado civil: Soltero Casado Unión libre

Si es padre o madre de familia ¿cuántos hijos tiene?

Estudios del encuestado: Escuela Colegio Universitario

Nivel de ingresos mensual: $\quad$ Menos de 1 salario mínimo (\$616.000)

Más de 1 salario mínimo

Religión: Católica Protestante Otra: ¿cuál? ¿Por cuál medio se moviliza más frecuentemente? Caminando bicicleta bus carro propio 
A1. Conocimiento sobre los factores que contribuyen al calentamiento global (cambio climático)

Pregunta: A continuación encuentra una lista de aspectos que pueden, o no, contribuir al calentamiento global (cambio climático). En Santa Marta, ¿cuáles de las siguientes acciones estarían contribuyendo al cambio climático?

\begin{tabular}{l|c|c|c|c|}
\hline 1. Las centrales eléctricas & Si & $X$ & No & \\
\hline 2. Los carros y vehículos de transporte & Si & $X$ & No & \\
\hline 3. El uso de espray de aerosol & Si & & No & $X$ \\
\hline 4. El uso de plaguicidas & Si & & No & $X$ \\
\hline 5. El suministro y consumo de agua potable & Si & $X$ & No & \\
\hline 6. El uso de aparatos eléctricos como congeladores, televisores, aires & Sí & $X$ & No & \\
acondicionados, etc. & & & & \\
\hline 7. El agujero de la capa de ozono & Sí & & No & $X$ \\
\hline 8. Los teléfonos celulares & Si & & No & $X$ \\
\hline
\end{tabular}

Las casillas marcadas corresponden a la respuesta correcta. Se asignó un punto para las respuestas correctas; se restó un punto para las respuestas incorrectas.

\section{A2. Indice de percepción de riesgo}

Pregunta: ¿Qué tan probable cree usted que las siguientes situaciones ocurrirán durante los próximos 50 años debido al calentamiento global (cambio climático)?

\begin{tabular}{l|l|l|l|l|l|}
\cline { 2 - 6 } & No & Poco & No & Algo & Muy \\
probable & probable & sabe & Probable & probable \\
\hline $\begin{array}{l}\text { 1. En todo el mundo, el nivel de vida de mucha } \\
\text { gente disminuirá debido al cambio climático }\end{array}$ & & & & & \\
\hline $\begin{array}{l}\text { 2. En todo el mundo se producirá escasez de } \\
\text { agua como consecuencia del cambio climático }\end{array}$ & & & & & \\
\hline $\begin{array}{l}\text { 3. En todo el mundo las tasas de enfermedades } \\
\text { graves aumentarán debido al cambio climático }\end{array}$ & & & & & \\
\hline $\begin{array}{l}\text { 4. Su nivel de vida o el de su familia disminuirá } \\
\text { debido al cambio climático }\end{array}$ & & & & & \\
\hline $\begin{array}{l}\text { 5. En Santa Marta habrá escasez de agua como } \\
\text { consecuencia del cambio climático }\end{array}$ & & & & & \\
\hline $\begin{array}{l}\text { 6. La posibilidad de que usted o su familia se } \\
\text { enfermen de algo grave aumentará como } \\
\text { consecuencia del cambio climático }\end{array}$ & & & & & \\
\hline
\end{tabular}




\section{A3. Conocimiento sobre los efectos en la salud por causa del calentamiento global (cambio climático)}

La siguiente lista contiene algunas enfermedades que pueden estar afectadas por el cambio climático, mientras que otras no. ¿Cuáles de las siguientes enfermedades considera que están siendo afectadas por el cambio climático?

\begin{tabular}{l|c|c|c|c|}
\hline 1. Las enfermedades infecciosas, como el dengue o la malaria & Si & $X$ & No & \\
\hline 2. El asma y las afecciones respiratorias (gripe, resfriado, etc.) & Si & $X$ & No & \\
\hline 3. Alergias & Si & & No & $X$ \\
\hline 4. Afecciones cardiovasculares & Si & $X$ & No & \\
\hline 5. Las infecciones que pueden causar la diarrea & Si & $X$ & No & \\
\hline 6. El cáncer de piel & Si & & No & $X$ \\
\hline 7. Las olas de calor (insolación ylo deshidratación) & Si & $X$ & No & \\
\hline
\end{tabular}

Las casillas marcadas corresponden a la respuesta correcta. Se asignó un punto para las respuestas correctas; se restó un punto para las respuestas incorrectas.

A4. Acciones políticas frente al calentamiento global (cambio climático)

El gobierno municipal tiene un papel importante para garantizar que Santa Marta reduzca su impacto en el clima. ¿Hasta qué punto usted está de acuerdo o en desacuerdo con las siguientes medidas?

\begin{tabular}{|c|c|c|c|c|c|}
\hline & $\begin{array}{l}\text { Totalmente } \\
\text { en } \\
\text { desacuerdo }\end{array}$ & $\begin{array}{l}\text { Parcialmente } \\
\text { en desacuerdo }\end{array}$ & $\begin{array}{l}\text { No } \\
\text { sabe }\end{array}$ & $\begin{array}{l}\text { De } \\
\text { acuerdo }\end{array}$ & $\begin{array}{l}\text { Totalmente } \\
\text { de acuerdo }\end{array}$ \\
\hline \multicolumn{6}{|l|}{$\begin{array}{l}\text { 1. Invertir recursos en proyectos de } \\
\text { energía limpia como los parques eólicos, } \\
\text { podría satisfacer la demanda de energía } \\
\text { con el uso del viento }\end{array}$} \\
\hline \multicolumn{6}{|l|}{$\begin{array}{l}\text { 2. Invertir recursos en mejorar la } \\
\text { información y educación sobre cambio } \\
\text { climático en Santa Marta }\end{array}$} \\
\hline \multicolumn{6}{|l|}{$\begin{array}{l}\text { 3. Invertir recursos en subsidiar } \\
\text { elementos como los paneles solares }\end{array}$} \\
\hline \multicolumn{6}{|l|}{$\begin{array}{l}\text { 4. Aumentar los impuestos a los } \\
\text { combustibles para que las personas } \\
\text { utilicen menos sus carros }\end{array}$} \\
\hline \multicolumn{6}{|l|}{$\begin{array}{l}\text { 5. Colocar un impuesto a las empresas } \\
\text { que utilicen la energía de forma } \\
\text { ineficiente, aunque esto derive en } \\
\text { aumento de los precios de los productos }\end{array}$} \\
\hline $\begin{array}{l}\text { 6. Invertir recursos en un sistema de } \\
\text { transporte público que sea más } \\
\text { eficiente, para que las personas utilicen } \\
\text { menos sus carros }\end{array}$ & & & & & \\
\hline
\end{tabular}




\section{A5. Indice de la voluntad para actuar}

Pregunta: ¿Hasta qué punto usted estaría dispuesto a hacer algo de lo siguiente?

\begin{tabular}{l|l|l|l|l|c|}
\hline & $\begin{array}{c}\text { NO } \\
\text { rotundo }\end{array}$ & $\begin{array}{c}\text { Probablemente } \\
\text { No }\end{array}$ & $\begin{array}{c}\text { NO } \\
\text { sabe }\end{array}$ & $\begin{array}{c}\text { Probablemente } \\
\text { SI }\end{array}$ & $\begin{array}{c}\text { SI } \\
\text { rotundo }\end{array}$ \\
\hline $\begin{array}{l}\text { 1. Comprar aparatos de bajo consumo } \\
\text { de energía: bombillas, automóviles }\end{array}$ & & & & & \\
\hline 2. Utilizar menos el aire acondicionado & & & & & \\
\hline 3. Comprar paneles solares & & & & & \\
\hline $\begin{array}{l}\text { 4. Compartir el carro por lo menos un } \\
\text { par de días a la semana }\end{array}$ & & & & & \\
\hline $\begin{array}{l}\text { 5.Usar el transporte público al menos } \\
\text { dos veces por semana }\end{array}$ & & & & & \\
\hline $\begin{array}{l}\text { 6. Ir a pie o en bicicleta al trabajo, de } \\
\text { compras o a otros lugares }\end{array}$ & & & & & \\
\hline
\end{tabular}

